Voix et Images

\title{
Jacques Godbout romancier : Le voyage, le dragon et l'Amérique
}

\section{André Renaud}

Volume 5, numéro 1, automne 1979

Jacques Godbout

URI : https://id.erudit.org/iderudit/200186ar

DOI : https://doi.org/10.7202/200186ar

Aller au sommaire du numéro

Éditeur(s)

Les Presses de l'Université du Québec

ISSN

0318-9201 (imprimé)

1705-933X (numérique)

Découvrir la revue

Citer ce document

Renaud, A. (1979). Jacques Godbout romancier : Le voyage, le dragon et l'Amérique. Voix et Images, 5(1), 5-32. https://doi.org/10.7202/200186ar d'utilisation que vous pouvez consulter en ligne.

https://apropos.erudit.org/fr/usagers/politique-dutilisation/ 


\section{Jacques Godbout romancier: Le voyage, le dragon et l'Amérique}

\section{DE LA MOUTARDE ET DU JAUNe D'CEUf A LA MAYONNAISE}

J'ai rencontré Jacques Godbout, le 4 juillet (1979), à l'Office National du Film, à Montréal. Nous avions convenu, lui et moi, d'une rencontreconversation très simple dont le thème devait être unique, les ramifications indéfinies. Une part de systématique, une part d'inattendu. Je voulais depuis longtemps discuter avec l'auteur de Salut Galarneau de l'errance, des pérégrinations de ses personnages. Cela est donc devenu le thème de notre entretien. Faut-il dire que je ne cherchais pas ainsi à circonscrire ce qui m'eût paru être le thème principal des cinq romans de notre auteur. Non pas! J'avais depuis plusieurs années retenu celui-là pour son intérêt dans une littérature romanesque toute remplie d'exilés, d'évadés, de déserteurs, de voyageurs, d'engagés, et où l'ailleurs devient l'antithèse et la problématique de l'ici plutôt que d'en être la prolongation dans l'espace et dans le temps. Je ne recherchais qu'une seule des multiples analyses. Je trouvais donc que dans une écriture très actuelle, Jacques Godbout demeurait - comme ses prédécesseurs - préoccupé par le pays natal mis en opposition avec la géographie terrestre et globale, avec les géographies européenne et étatsunienne en particulier. Cela me suffisait ! Car le romancier d'ici est un errant, comme le Canadien de la chanson. II est beaucoup plus, bien sûr! Mais il est survenant, souci de venir, de revenir, de se souvenir. II est souvenance et, souvent, aliénation, c'est dire sans souvenance, à la recherche de son temps... perdu. Sans le savoir, il est proustien.

Ainsi j'ai lu les romans de Jacques Godbout, toujours. Comme j'avais lu auparavant tous ceux d'ici, car, malgré la différence des styles et des esthétiques, ils se ressemblent tous. Depuis le Chercheur de trésors jusqu'au "Chasseur de Dragons". La rencontre s'est déroulée en deux temps: l'un officiel durant l'enregistrement (une heure et demie), l'autre officieux à table au déjeuner (une heure et demie). Nos romanciers mangent aussi, comme les Frères de mon instruction chrétienne faisaient pipi, ce que j'ai découvert bien tard dans ma vie à cause de la soutane. En quoi consiste donc cet entretien, sinon à présenter ici un auteur en pleine réflexion sur ses personnages, donc 
sur lui-même. Comme de vrais philosophes, nos romanciers vivent pour être capables d'écrire et de penser. Sur le menu fretin ils ont l'avantage combien énorme - d'analyser à haute voix ou à voie écrite leur être en gestation. Cela, en fin du compte, donne un roman... et deux et plus si le courage est là comme soutien. II n'y a donc ici aucun système reconnu; il y a une circonstance. Si son expectative allait plus loin, le lecteur sera déçu. Le titre que j'ai choisi pour ce texte était inattendu. C'est Jacques Godbout luimême qui l'a spontanément proposé, au fil de notre conversation, sans même savoir que j'allais en faire un titre. Peu importe!

Jacques Godbout demeure notre romancier le plus immédiat, très rapproché du journalisme, du chroniqueur, dans les sens nobles de ces deux mots.

Ceux de sa race ne sont-ils pas les meilleurs romanciers de l'Histoire? La littérature se fait, je l'ai maintenant appris pour ne plus jamais l'oublier, au cœur même du quotidien.

ROMANS PUBLIÉS PAR JACQUES GODBOUT:

L'Aquarium, Paris, Éd. du Seuil, 1962, $156 \mathrm{p}$.

Le couteau sur la table, Paris, Ed. du Seuil, 1965, 157 p.

Salut Galarneau, Paris, Éd. du Seuil, 1967, 154 p. (Traduit en anglais par Alan Brown. Hail Galarneau, Don Mills, Ontario, Longmans, 1970, 131 p.).

D'Amour, P.Q., Montréal, HMH, 1972 et Paris, Ed. du Seuil, 157 p.

L'is/e au dragon, Paris, Ed. du Seuil, 1976, 158 p.

\section{NOTES POUR UNE RENCONTRE AVEC JACQUES GODBOUT}

Mener toute l'interview dans le sens de la géographie, dans le sens physique, puis dans le sens anthropologique du mot. Cela veut dire que lorsqu'il se déplace sur le globe terrestre ou sur le continent, le personnage cherche à mieux comprendre sa géographie natale, soit en la rejetant, soit en sentant le besoin de la reconquérir. Il y a surtout au début de l'œuvre romanesque déplacement physique, c'est-à-dire changements de lieux de vie. Plus tard, les déplacements se feront moins grands dans l'espace réel, tout aussi importants sans doute dans l'espace vital. Ce que je veux dire ici, c'est qu'entre les voyages du personnage innomé de l'Aquarium et ceux de Mireille et Mariette, par exemple, il n'y a pas de commune mesure sinon celle de l'orinisme ou de la fantaisie. Dans toute cette géographie humaine, il y a des tentatives d'exil, de désaffectation, de réappropriation du pays natal. C'est la question existentielle de l'être québécois qui se trouve dès lors posée et à laquelle les personnages, selon les romans eux-mêmes, et dans l'économie même de ceux-ci, essaient de répondre au gré des circonstances de leur vie mouvementée. 
J'aimerais que notre conversation, sans être rigide ni trop académique, suive l'ordre, la chronologie des romans, depuis L'Aquarium jusqu'à L'isle au Dragon. Il y a là plus de quinze ans d'écriture, donc d'observation puis de réflexion sur l'être et le devenir québécois. Il est bien entendu que se greffent à mon concept de géographie, de voyages, départs, exils, retours, tous les autres éléments qui en fait composent la vie de l'individu, depuis ses réactions face à son pays, à la société où il a été malgré lui propulsé par l'accident même de sa naissance jusqu'à son attitude par rapport aux siens, par rapport aux autres, tout autant dans sa vie de couple que dans sa vie collective. Entre les destinées personnelles et singulières des personnages et le regard de l'écrivain sur le devenir québécois, il y a des relations privilégiées dont il faudrait aussi discuter. Notre propos ouvre donc toutes grandes ouvertes les avenues de l'homme dans son expression totale, natale, sociale, linguistique, familiale, politique. J. Godbout traite de l'homme américain français dans ses multiples efforts de connaissance de lui-même, de reconnaissance, de ré-appropriation, tantôt ardue et tantôt jubilante de son destin.

\section{L'Aquarium (1960)}

Je vous propose ici, tel que je le perçois, un portrait du personnage innomé de l'Aquarium, Canadien français (non pas Québécois), gratuitement intellectuel, sans famille ni filiation sentimentale. II voit son pays comme une immensité blanche et froide. Il est solitaire, un peu superbe, appartenant, si le mot est convenable, à une espèce d'univers cosmopolite, ésotérique, ayant jadis fait un rêve colonialiste, lui-même colonisé et aliéné. C'est un déchu, un peu comme celui du célèbre poème d'Alfred Desrochers, mais sans doute encore davantage.

Devenu inutile, il se fait égocentrique et trouve son salut, ou plutôt sa fuite grâce à ce merveilleux personnage qui s'appelle Lui, véritable image du Rédempteur sacrifié et réincarné dans le personnage non moins beau d'Andrée (qui signifie force virile, qui est une femme et qui devient la corédemptrice en quelque sorte. Ce personnage vit une expérience à trois volets :

a) son antériorité canadienne qui est creuse comme un tambour; b) son exil infructueux (sauf pour ce qui est de la présence de l'autre); c) sa réjuvénation auprès d'Andrée, réjuvénation qui fait de lui un éternel apatride trouvant son propre salut vers New York, ou peut-être éventuellement vers l'Europe, mais rien n'est déterminé si ce n'est cette fuite toute personnelle qui, toute comparaison étant odieuse, rappelle quand même celle d'Hervé Jodoin du Libraire de Bessette.

II ne lui reste plus qu'à assurer son bien-être financier, qu'à troquer la langue française pour l'anglaise et qu'à vivre heureux jusqu'à la fin de sa vie. En somme, c'est un personnage qui n'a plus, qui ne veut plus d'appartenance. C'est le déserteur, mais non pas comme l'entendait Damase Potvin, Claude- 
Henri Grignon, ou Louis Hémon. C'est le déserteur impuni! Ce en quoi il se différencie - du moins dans ce premier roman - de ses prédécesseurs auxquels je fais ici une mention bien délibérée. C'est un Survenant qui n'a pas encore envie de revenir, contrairement au sublime personnage de Germaine Guèvremont.

\section{Le couteau sur la table (1963)}

Dès l'abord, le personnage du Couteau sur la table, lui aussi sans nom et sans famille, donc sans filiation générique, se présente comme un déserteur, dans le sens natal et militaire du mot.

Dans le sens linguistique, ce qui me paraît être une nouvelle dimension dans l'œuvre. Dimension qui apparaît, s'imprègne et qui ne la quittera, je pense, que dans $L$ '/sle où la langue est acquise plutôt que d'éclater comme feux d'artifices, d'abord dans Salut Galarneau, puis ensuite dans D'Amour P.Q.

Mais ne retenons pour l'instant que le très révélateur itinéraire dù personnage du Couteau. C'est lui aussi un exilé, mais au Canada, dans les Plaines de l'Ouest, où il affirme une filiation de dépendance totale vis-à-vis d'une femme anglophone, jeune, jolie, riche. C'est ici que commence vraiment - de façon dramatique et c'est le moins que l'on puisse dire - à paraître les déchirements entre l'anglophilie et l'anglophobie, entre l'aliénation et la dure appartenance à l'américanité ou, plus précisément, à "l'American Way of Life".

Dans le sens de notre propos, je crois que Patricia compose à elle seule une géographie; elle-même expatriée (mais jamais apatride) elle représente et incarne une aire d'utopie. Plus qu'Andrée, elle est charnelle, moins abstraite que la première qui pourtant n'est pas sans être charnelle. Patricia marque à la fois l'accession à un rêve du dominé enfin dominateur et qui n'est jamais cependant acquis à sa domination, à cause de sa dépendance matérielle, à cause bien davantage d'un profond sentiment - nouveau dans l'œuvre - de la nostalgie du pays natal, voire de la trahison.

Le narrateur du premier roman n'a jamais, à mon avis, ressenti cette dure dualité. La fuite vers New York ou vers l'Europe lui paraissait acceptable et viable.

Le personnage du Couteau ressent autre chose de plus primordial qui s'appelle tout simplement le retour à la source vitale. D'où ce magnifique voyage en train qui fait que le pays natal est reconquis à ras de sol, lentement, kilomètre après kilomètre. Dès lors se manifestent dans l'œuvre, et pour la première fois, la dualité et l'ambivalence de Montréal, bastion anglais et terre québécoise. Deux exilés rentrent ainsi chez eux, liés par l'amour physique et séparés par l'histoire. Et c'est le sens, la conscience de l'historialité qui l'emporteront sur un premier sentiment qui d'ores et déjà n'a, pour ainsi dire, plus aucun sens. Dès lors, il faut parler de Madeleine; et l'on ne saurait évoquer l'image et le rôle de ce personnage sans rappeler Andrée... 
Andrée, je parle ici du personnage ontologique, ne semble avoir d'épaisseur que dans la mesure où elle renvoie à Lui où elle représente quelqu'un d'autre, où elle est émissaire. On dirait qu'elle est miraculeuse, salvatrice. Je vous avoue que je ne puis penser à Lui sans penser au Christ ni à Madeleine, sans penser à Marie, espèce de personnage de chair, certes, mais aussi personnage ascendant, transcendant. Madeleine, c'est dans l'œuvre la naissance ou disons l'apparition de la Québécoise: réelle, vivante, pratique, amante et génitrice, encore qu'on la fasse mourir pour la trés simple raison que le temps n'est pas venu, à ce moment-là de l'œuvre, de faire s'éclore la famille, elle qui viendra si merveilleusement dans le troisième roman, c'est-à-dire dans Salut Galarneau! Bien sûr, Madeleine a une sœur et des parents, mais c'est sous le signe de la mort qu'on les voit ensemble, l'espace de quelques minutes seulement.

Et dès après, cette vue embryonnaire et comme infructueuse de la famille sera réduite à nouveau à l'individualité lorsque le narrateur fera l'amour avec la sceur de Madeleine, en une des plus belles scènes du roman.

Tous ces épisodes se déroulent à Montréal, une ville d'ores et déjà marquée par les bouleversements de la révolte, une ville qui fascine le narrateur et qui, pourtant ne sait pas le retenir.

Avec l'astuce du personnage de l'Aquarium, celui du Couteau prépare sa fuite (on songe encore ici à Jodoin) qu'il réussit en maître fuyard. C'est la deuxième fois qu'il part, qu'il gagne l'exil selon l'expression consacrée ici, n'ayant su découvrir dans sa terre natale aucune de ses attaches essentielles qui font une patrie. Et encore une fois la voie s'ouvre vers les États-Unis qui ont été par excellence la terre d'exil, la Klondike, l'utopie des Canadiens français depuis les premières grandes migrations de la deuxième moitié du dix-neuvième siècle. Tous nos romanciers prédicants, je ne pense pas à Godbout bien sûr, mais à des gens comme Potvin et Grignon pour ne mentionner que ceux-ci, tous nos romanciers prédicants ont dépeint les États-Unis comme une terre de facilité, de misère, parfois de débauche, où l'on suffoque dans la fumée malodorante et polluée des grandes usines. Images dantesques qui font aujourd'hui sourire mais, faut-il le dire, qui ne sont pas sans préfigurer toutes celles, nombreuses et beaucoup mieux faites des romans même de Jacques Godbout où l'on fait la satire et la parodie à la fois de la société américaine étatsunienne, oủ l'on décrit tous les maux qu'elle a engendrés et qui sont d'ores et déjà devenus des signes de civilisation: les sacrifices, les détergents, le Pepsi et je ne sais combien d'autres agents du même acabit qui, mis ensembles, font le portrait de la déchéance humaine. Mais cela se produit surtout dans le troisième roman et nous sommes ici dans le Couteau.

J'aimerais enfin revenir au cheminement, à l'errance du héros du second roman pour conclure. Après plusieurs années donc d'un exil qui n'est pas davantage analysé, qui est comme un espace silencieux dans le roman, le personnage revient auprès de Patricia. Ce retour, et plus précisément ce retour vers la femme anglophone des premières amours, sera interprété de 
plusieurs façons, dont je retiens les suivantes. Ou bien le personnage vient ici accomplir l'holocauste qu'il aurait dû réaliser beaucoup plus tôt dans sa vie, au moment où il avait quitté Patricia pour Madeleine. C'est à cette époque-là qu'il aurait eu avantage à tuer sa première maîtresse, c'est-à-dire à faire mourir en lui-même jusqu'au souvenir le plus concret de ses filiations au monde anglophone. Ou bien le personnage rêvera de tuer Patricia et n'y arrivera pas, faute de courage ou par respect de la vie.

Le dénouement, la fin, la dernière séquence de ce roman nous paraîtra importante dans la mesure même où elle est problématique, hypothétique, à l'image du personnage dont Patricia aura à quelques reprises déclaré qu'il est faible et assez peu entreprenant malgré une faconde et une superbe de surface. Le second roman se termine donc un peu comme le premier, dans une aire d'existence théâtrale et, je viens d'employer le mot, à la fois problématique, peut-être même tout à fait onirique.

\section{Salut Galarneau (1967)}

C'est le roman de l'éclatement géographique dans le sens anthropologique du mot. Je pense que c'est le moment privilégié de la série où le personnage devient Québécois américain, après avoir été Canadien français tout pur dans l'Aquarium et Canadien français puis Canadien français québécois dans Le Couteau sur la table.

Mais commençons donc par le commencement, c'est-à-dire par le titre même du roman qui marque ou proclame deux phénomènes complémentaires. D'abord la salutation du premier mot que j'interprète ici comme étant une reconnaissance réciproque auteur-personnage, comme une espèce de baptême grâce auquel s'annoncent la naissance et la fraternité, ou la paternité enfin assumée par la salutation, par l'acceptation. Cela est suivi immédiatement et c'est une première dans l'œuvre de Jacques Godbout par l'annonce du nom de famille Galarneau, également première puisque c'est le personnage de la famille que l'on arrête sur une consonance québécoise, tout à fait celle de Galarneau. Bien sûr, pourriez-vous m'opposer, il y avait déjà dans l'Aquarium, le nommé Lauzon! Mais Lauzon, personnage secondaire s'appelait Lauzon comme on s'appellerait Faure ou MacDonald, c'est-à-dire quelqu'un qui ne conserverait de ses attaches premières que le très lointain souvenir d'une vague appartenance qui, d'ailleurs, n'importe à peu près plus. Ici, c'est la juxtaposition sémantique des deux mots qui semble compter, dans la mesure même où le nom Galarneau est précédé par l'interpellation salutaire, donc fraternelle ou, pour le moins amicale et entendue. Mais revenons à notre propos qui est de suivre le personnage dans sa mouvance, d'autant que le titre de ce troisième roman me semble signifier beaucoup plus puissamment à la fin du roman qu'à son début. C'est dire en fin de compte que ce titre suit le roman plutôt qu'il ne l'annonce ou l'enveloppe. Cela n'est pas un titre-somme, comme Trente Arpents, par exemple, ni un titre anecdotique, comme The Tin Flute tel qu'on a superbement traduit Bonheur d'occasion. Nous reviendrons donc là-dessus plus loin durant notre interview. 
Je souhaiterais retrouver dès maintenant François Galarneau, en notant au passage son patronyme proche des mots Français et France. François me paraît être le roi, le roi déchu, peut-être bien, mais celui qui cherche à assumer sa déchéance en un pays lui-même déchu, mais dont il essaie de reconstituer la vieille noblesse par son propre voyage à l'intérieur de ses petites frontières et par le voyage de l'autre, cette fois-ci par le voyage d'un frère, vers et dans la mère-patrie, ce frère adulé s'appelant Jacques, comme l'auteur lui-même. La route de François Galarneau, inscrite sous le signe de la famille, du clan, donc d'une antériorité acceptée, elle est double, parallèle et complémentaire. La sienne propre d'ailleurs, depuis Montréal jusqu'à Lauzon, en face de Québec, puis de retour vers Montréal après une première filiation conjugale qui n'est pas sans rappeler le premier couple avorté (Madeleine et le narrateur, personnages principaux du Couteau sur la table). François ne saura expliquer ni justifier les premières expériences de son âge adulte que grâce au séjour européen de son frère Jacques dont il envie non seulement la chance et l'érudition éventuelle, mais surtout la possibilité énorme de voir, de vivre, de co-naître dans le sens claudélien du mot.

II me semble que par procuration, par l'intermédiaire du frère aîné Jacques, François aura fait lui-même son profond pèlerinage aux sources natales, en France. C'est dire qu'il aura suffisamment réfléchi sur l'aventure de l'aîné pour en retirer tout le profit personnel.

N'oublions pas que dans ce roman, Jacques n'aura joué un rôle primordial que pour mieux s'estomper, puis s'effacer dans la mesure où il est d'ores et déjà devenu insignifiant. C'est François en réalité, qui se trouvera libéré par ce voyage dans le vieux pays, parce que c'est lui qui s'affranchira et qui se proclamera lui-même roi! Roi du Hot Dog! D'accord! Mais roi quand même, de son destin, de sa promotion intellectuelle et existentielle. Le célèbre « nowhere" du samedi soir me paraît ici important, non pas tant parce que Marise entre dans la vie de François, mais bien parce que Marise (Marie, Madeleine) propose une liberté d'expression individuelle, une maturité conquise, une solitude en quelque sorte rassérénante.

Auprès d'elle François vit sans problème, sans l'énorme problème que soulevait par exemple le personnage de Patricia auprès du héros du Couteau. C'est elie qui permet l'édification de la boîte aux Hot dogs et aux patates frites, cette dramatique installation aux bords de la route, donc sur la route qui relie les villes d'ici, et en face du fleuve, donc sur la mer qui mène d'ici aux vieux pays. Se rejoignent dès lors le présent de François et son histoire, l'instant et l'historicité. C'est la première fois que cela se produit dans l'œuvre, c'est-à-dire, la simultanéité du présent et de l'Histoire. Le goût d'écrire, disons le besoin impérieux d'écrire vient à François de cette double conjoncture, beaucoup plus que de ses loisirs, car, quoiqu'il en dise luimême, quoiqu'il pense de lui-même, ce personnage est le plus fébrile de tous (jusqu'ici).

Pourquai François écrit-il sinon pour retracer son chemin qui ne peut plus se dissocier de celui de ses frères, de celui de sa mère, de toute sa 
famille, de sa collectivité. Cet étrange itinéraire accepté le mènera à l'initiation baptismale dans les eaux du fleuve. Son exercice d'écriture le mènera aussi au silence et à l'emmurement, deux phénomènes dont il faudra tenir compte.

\section{D'Amour P.Q. (1972)}

On pourrait dire de Mariette et de Marielle qu'elles voyagent à l'inté rieur même de leur appartement, qu'elles y ont d'ores et déjà trouvé une aire d'existence suffisante et qu'elles y vivent un destin marqué aux signes de la joie et de la bonne santé. Elles ont, avant même que ne commence le roman, assumé leur être québécois, leur identité, leur langue, leur corps, surtout leur corps, superbement ébloui et totalement consenti. C'est la première fois dans l'œuvre romanesque que des personnages - et cette fois-ci ce sont deux femmes ou plutôt un couple de femmes - semblent vouloir afficher une espèce d'indolence qui frise l'inconscience et qui pourtant n'en est pas tout à fait. D'Amour $P$.Q. se présente comme étant une fête, une célébration, un rite de la liberté. Ici dès le générique les personnages sont pour ainsi dire baptisés et entendent décréter jusque dans le détail leur droit à la vie sans questions, sans recherche métaphysique.

Et pourtant, cela n'est pas juste, car elles seront elles aussi itinérantes, dans la mesure même oủ elles auront à confronter un autre univers, celui de la fiction, celui de l'utopie et du rêve, le monde lointain et écrasant, frustrant, de la littérature. C'est comme si l'auteur, grâce à elles deux, mettait son propre cheminement d'écrivain à dure épreuve en créant cet étrange personnage loufoque qui s'appelle Thomas D'Amour, qui s'installe theâtralement à l'envers du réel, à contre-courant d'une si simple réalité qui se trouve être celle de ces deux jeunes femmes pétillantes qui ne demandent rien d'autre que de passer dans la vie en obéissant sans arrière-pensée aucune aux mille fantaisies d'une belle jeunesse. Marielle et Mariette désorganisent l'œuvre romanesque et, pourtant, elles s'inscrivent très bien en son cours. Elles parachèvent, à mon sens, la recherche entreprise par François Galarneau et par ses prédécesseurs en rabaissant l'acte d'écrire à une préoccupation très concrète, ou plutôt en découvrant à la fiction des obligations matérielles qui passent par la grammaire, le lexique, la ponctuation et, surtout par le doute, par ce carrefour inoui où l'imagination se heurte à la réalité de la faim, de l'envie de prendre un bain et de l'envie de s'adonner aux plaisirs de l'amour. II y a ici une entreprise de démystification qui attaque l'acte du romancier et qui, du même coup entend redorer le blason de l'écrivain. Nous sommes en coulisses, à l'envers du décor, là où l'écrivain-vedette perd son vernis au profit de l'écrivain laborieux, là où le lecteur joue le premier rôle parce qu'il peut tout à loisir, non pas seulement détruire l'écriture, mais la refaire, la reconstituer selon ses propres désirs.

Thomas propose aux deux jeunes femmes des aventures écrites que vient détruire la vie elle-même, qu'elle contrecarre à chaque phrase tant et si bien que la littérature elle-même se transforme en une sorte d'a-littérature, 
que le héros devient anti-héros, démuni et dénudé, réduit aux dimensions risibles et ridicules du signe phallique.

Avec D'Amour P.Q. le héros masculin est réduit à ses seules possibilités oniriques, à ses seuls phantasmes. Ici la femme seule et splendide peut dépasser l'ordre historique et social. Elle n'a plus besoin d'espace, ni de géographie, qui ne soient ceux qu'elle a elle-même choisis sans consultation, sans domination. Je pense qu'à première analyse, il pourrait paraître difficile de situer ce quatrième roman dans la géographie de l'ensemble, tellement sont transformées les propositions des trois premiers romans. Parce que les préoccupations des autres personnages s'inscrivent dans la voie énigmatique, dans l'avenue difficile où la réalisation de soi-même exige un "questionnement" de son pays natal, un rejet puis un retour, un exil puis une pénible ré-appropriation non moins douloureuse. Rappelons-nous avec combien de sacrifices François Galarneau a réussi l'ceuvre de sa "salvation" personnelle, de son beau retour à sa terre puis à son milieu familial, ayant enfin appris, je crois, à s'aimer lui-même, corps et âme. Ce long processus, qui en quelque sorte compose tout le troisième roman, me paraît déjà accompli dans la phase pré-écrite de D'Amour P.Q. Descendantes, bien sûr, de François, Marielle et Mariette ont assumé leur être québécois et n'ont plus à se poser là-dessus aucune question d'ordre philosophique ou d'ordre existentiel.

Québécoises, elles existent en tant que telles, consommatrices averties, utilisatrices de shampooing, de postes-radios, de télé; mangeuses de sandwiches et de hot-dogs, rouges de catchup. Elles n'ont plus besoin de voyager en Amérique puisque I'Amérique se résume en elles, puisqu'elles sont devenues l'Amérique et, en somme, l'américanité même. Si elles contestent Thomas, c'est qu'il leur propose Dieu sait quelle contemplation devenue insignifiante. Ces filles ont une filiation profonde avec le réel et elles entendent être fidèles, corps et âme à ces racines.

C'est sans doute pourquoi elles refont de toutes pièces le roman de Thomas, c'est pourquoi elles lui dictent une littérature mieux adaptée à leur goût puis à leur américanité, c'est-à-dire celle de la bande dessinée, celle du super-héros (du superman), contraire du héros et de l'anti-héros dans les sens traditionnels, j'allais dire classiques, des termes.

Si François Galarneau (qui est le plus beau personnage) était allé plus loin, s'il avait vécu davantage, il serait devenu, lui-même, ce double personnage de Marielle-Mariette. Si François voyait ces deux jeunes femmes, je suis sûr qu'il deviendrait leur parrain ou leur caution morale, tellement il aurait souhaité lui-même accomplir leur voyage, leur voyage dans la vie réelle et dans la vie de l'écriture.

\section{L'Isle au Dragon (1976)}

II reviendra au personnage du cinquième roman, ce gout du voyage. II s'agit cette fois de Paris et New York, l'Europe et l'Amérique constituant 
encore ici les deux pôles vitaux, comme pour les autres romans, si l'on exclut $D$ 'Amour P.Q. où l'on est exclusivement québécois, oủ l'on ne se déplace que dans l'utopie de la littérature. Le personnage de l's/e poursuit lui aussi un monde utopique dans la mesure oủ son île est ouverte aux quatre vents, s'épanouit sur les océans, s'offre à l'établissement de relations avec l'univers. Mais ce personnage, lui-même très problématique, poursuivra un dialogue de sourd, en quelque sorte, puisque ses invitations restent lettres mortes.

Michel Beauparlant - l'auteur a sûrement choisi son nom avec circonspection - se présente aux lecteurs comme étant un insulaire, lui-même dragon, qui entreprend une campagne de protection de l'environnement contre l'invasion du capitaliste barbare William T. Shaheen Jr. II s'agit d'assurer la pureté de l'air ambiant, partant d'assurer le devenir de l'aire vitale.

Encore ici, comme c'est le cas dans Salut Galarneau!, le héros se déplace par l'imaginaire et par objet interposé. II y a tout d'abord ces bouteilles qu'on lance à la mer et qui, chacune différente, portent des messages, lancent des invitations à la solidarité, à l'amitié des autres hommes. Michel Beauparlant ressemble au chevalier qui rêve de croisades et de conquêtes, de conversions du monde. C'est bien le cousin germain de François Galarneau, du personnage du Couteau, de celui de l'Aquarium, dans la mesure où la vie moderne - la société de consommation - l'a littéralement poussé à l'écœurement, dans la mesure surtout où il a conscience de se rebeller contre une gigantesque machine - contre la marche même de l'histoire - où il sait de façon aiguë qu'il n'est pas sûr qu'il va remporter la victoire.

II pourrait aisément y avoir une dimension tragique dans tous ces romans, disons plutôt qu'elle s'y trouve, qu'à certains carrefours le destin des personnages pourrait virer à la tragédie, que tous les éléments du tragique, de la victoire du Destin, de l'irrémédiable force de la Fatalité, tout se trouve là contenu. Et toute cette énorme puissance me paraît, non pas s'effondrer, mais être maîtrisée par la seule magie d'une écriture ironique. Le dragon, par exemple, ne pourrait être personnage tragique - comme dans Phèdre - que si son apparition était soutenue par une trame austère. Dès lors, il devient intervention directe du dieu ou de lui-même. De par sa perspective sur l'univers, de par son propre tempérament, le personnage godboutien est ironique, sarcastique, frondeur, caractéristiques à quoi se mêlent une grande sensibilité, un lyrisme et, sans doute, une vulnérabilité toute québécoise. II est, avec tout cela, assez puissant pour transformer des défaites en aiguillons et pour tenir tête au destin, selon l'expression populaire. C'est là sans doute l'explication à découvrir dans tous ces re-départs, ces recommencements qui marquent - sans paradoxe - les fins de romans. Le personnage de I'Aquarium s'enfuit avec Andrée vers New York ou vers l'Europe, et cette fuite, il faut bien l'interpréter comme une victoire sur une existence sombre et comme une ouverture sur la vie. A la fin du deuxième roman, l'aventurier appelle une ère nouvelle; des bombes éclatent qui sont signes de bouleversement, de révolution, donc de renouveau. François Galarneau, lui, regagne 
la société et déclare son intention de vivre. Marielle et Mariette s'en vont terminer par une communication téléphonique une aventure qui a débuté dans l'intimité de leur appartement. Quant à Michel, enfin, lui aussi vainqueur du dragon comme Saint-Michel, il écrase l'ennemi et reconquiert l'île menacée. Partout, donc, de nouveaux départs, la promesse de devenirs possibles, la conquête sur l'aliénation.

Sans doute ces personnages voyagent-ils parce qu'ils vivent dans un pays incertain, parce qu'ils ne se sont pas encore vraiment appropriès cet espace, ni ce temps difficiles.

Le voyage, c'est aussi une reconnaissance, la mission du co-naître, la quête du vrai, plus simplement le besoin de vivre. D'où l'importance du troisième roman, ou plutôt, le moment privilégié que représente l'investiture du troisème roman où, pour la première fois, nous avons insisté là-dessus, la narration proclame en le baptisant le nom du héros. Après Galarneau, il devenait loisible à l'auteur de s'adonner à des écritures plus fantaisistes, à des incursions plus ludiques, ce qui expliquerait D'Amour P.Q. et L'/sle au Dragon. 


\section{ENTREVUE AVEC JACQUES GODBOUT}

A.R. II y a toutes les méthodes modernes qui font qu'on peut trouver une signification à une œuvre littéraire. Si vous le permettez, j'aimerais suivre la méthode la plus simple, la plus spontanée, la plus "conversation entre nous", pour essayer de découvrir d'abord pourquoi votre personnage (et je pense au personnage du début, au tout premier personnage jusqu'à celui de L'/sle au dragon) a besoin de voyager, sent le besoin de se déplacer, de se trouver de nouveaux espaces, pourquoi il a besoin de s'exiler parfois, pourquoi il a besoin de fuir (parfois il s'évade, parfois c'est un fuyard). C'est un bonhomme qui bouge dans son espace, qui bouge depuis le personnage de l'Aquarium jusqu'au personnage de L'/s/e au dragon même s'il s'agit d'un espace insulaire.

II y a chez vous, dans les années 60 , ce personnage qui se présente à nous, qui est canadien et qui ne se nomme pas (on va parler aussi de l'identité des personnages, de leur appartenance à leur milieu familial, à leur Canada français puis ensuite à leur Québec). II y a donc ce personnage qui se présente et qui n'a pas de nom, qui vit dans un pays qui est plus ou moins bien identifié, dont on connaît le continent, dont on connaît l'atmosphère, un pays du Tiers-monde, qui se cherche luimême, qui, on le sent bien, s'est évadé d'un pays qu'il n'accepte plus ou qu'il n'arrive plus à circonscrire, qu'il n'arrive pas à bien définir sauf pour faire allusion à des espaces blancs, à une saison blanche, à l'hiver, au froid, et qui dans la solitude, dans une espèce de promiscuité avec d'autres personnages dont on verra tantôt la fausseté, dont on verra tantôt l'image ironique, va se découvrir un destin vague à la fin du roman, qui sera un destin Amérique, qui sera en quelque sorte après cet exil, après ce départ vers l'ailleurs, qui fera une espèce de retour vers une Amérique floue, symbolique, qui est celle de New York. Ce personnage n'est pas véritablement enraciné; on le verra revenir dans Le Couteau sur la table; mais je m'arrête ici pour l'instant. D'où lui vient donc ce besoin de s'exiler d'abord, et ensuite de se provoquer luimême, à l'intérieur de lui-même pour se découvrir peut-être une identité 
et ça grâce au personnage d'Andrée. Pourquoi votre personnage dès le début de votre œuvre voyage-t-il comme par un besoin vital?

J.G. II doit y avoir à cela plusieurs raisons génétiques. Personnelles. Littéraires. Si l'on m'offrait aujourd'hui d'aller dans la lune je n'hésiterais pas un instant. Comment peut-on ne pas voyager! Je ne connais rien de plus beau qu'un lever de lumière en pleine mer quand on a passé la nuit à mal dormir sur le pont d'un cargo. Je ne connais rien de plus agréable que de parcourir la place du marché d'un village inconnu. On voyage pour les stimuli. J'écris avec mon nez. J'ai besoin d'accumuler les odeurs. De comparer. Rembrandt a produit une œuvre énorme, sans jamais voyager. C'est qu'il faisait venir à Amsterdam et qu'il accumulait dans sa grande maison les objets du monde entier. Mes ancêtres étaient des chaloupiers. Je suis un enfant du fleuve et donc des chemins qui marchent. Ecrire c'est marcher.

Et puis nous habitons un pays qui n'a qu'un seul point cardinal : le nord. C'est impossible à vivre. II faut, pour l'équilibre, un sud. Une Floride. $Y$ voyager.

Sociologiquement puisque, comme le disait Mauriac, on n'est le romancier que d'une génération, disons que la mienne s'éveilla dans un Canada français des années '50 au moment où le clergé était à l'apogée de sa bêtise dominante. La pauvreté intellectuelle du pays était telle qu'il nous paraissait difficile de rester sur place pourrir sous le ciel de Duplessis. Il y avait une énorme brume sur le Québec.

Nous fréquentions les humanités gréco-latines. Normalement le collège classique préparait à la prêtrise: l'essentiel était le ciel. Aucun problème pour ceux qui avaient la vocation. Mais les autres? Sortis du cours classique il nous fallait aller ailleurs : il n'y avait rien autour de nous qui puisse répondre à notre curiosité aliénée.

Cela produisit les "retours d'Europe", de Jean-Éthier Blais à Hubert Aquin, et ça continue! Mais il fallait s'aérer. On ne pouvait penser dans une société qui censurait les livres et le cinéma, où tout était figé comme au plus profond de l'hiver.

Mais aussi je suis un impatient. Littéralement: je n'aime pas souffrir. Littérairement: je ne tiens pas en place. Le personnage de l'Aquarium avait besoin de la dimension physique et culturelle des États-Unis, de l'Europe ou de l'Afrique.

A.R. C'est intéressant de voir comment se situe le personnage dans l'Aquarium; comment il reproduit en quelque sorte ce qu'il a peut-être vécu, les traumatismes qu'il a peut-être vécus et s'en délivre du même coup. Le personnage de Monsignor, par exemple, et tous ceux qui l'entourent et tout ce qu'ils signifient, le personnage de Pauline, les autres personnes qui forment des couples plus ou moins heureux, plus malheureux qu'heureux je dirais, constituent tout ensemble et tous 
ensemble une espèce de monde dont il va se purifier grâce à deux personnages qui me paraissent extrêmement puissants et qui vont faire partie de son errance, qui vont faire partie de son cheminement.

Le premier personnage, personnage de la Rédemption à mon avis, qui s'appelle Lui, dont on ne connait pas l'identité, qui va s'enliser bêtement comme ça, et qui va mourir devant les autres qui ne feront rien.

Et un autre personnage qui va venir en deuxième partie. C'est le personnage d'Andrée et qui me paraît comme étant la co-rédemptrice en quelque sorte, la prolongation de cet autre personnage. Elle provient de Lui puisqu'elle a été sa maîtresse dans l'antériorité et revient comme par une espèce de miracle, bien sûr pour retrouver son amant qui n'est plus là, et devient ensuite la co-rédemptrice... Ce personnage de Lui, ce permier personnage dont la prolongation sera mieux incarnée et plus vivante dans le personnage d'Andrée, comment l'avez-vous conçu?

J.G. Mon dieu! Si l'on savait comment ces choses-là arrivent! Comment se forment soudain les personnages, à mesure que s'écrit la phrase et que se précise la structure! J'ai l'impression que comme dans les autres livres, les personnages principaux de l'Aquarium ne sont toujours que les deux facettes d'un même individu. Le "Je" de l'Aquarium ne va pas chercher tant à se purifier qu'à découvrir ce qu'il est, dans «La Casa occidentale", qu'il devra quitter dès qu'il saura. Mais c'est le même mouvement qu'a suivi l'autre ("Lui») qui cherchait à donner, à se sacrifier, à agir dans une société dont il désirait la transformation profonde. Or les "révolutions" se pensent toujours à l'étranger, mais ne peuvent se réaliser que chez soi. "Lui" et le "Je" ne sont que deux facettes d'une même démarçhe. L'un est le désir de l'autre, si vous voulez. Et, probablement, si on prend Galarneau, François et Jacques, l'un est aussi le désir de l'autre. Ce sont les deux possibles d'un même personnage.

A.R. Le frère Jacques voyageant pour François. Ce n'est pas François qui est allé en Europe.

J.G. Jacques y est allé pour François. D'ailleurs il lui a envoyé des lettres, des cartes postales, et le reste. II n'y a donc pas nécessité qu'il s'y rende puisque voyager c'est aussi vivre dans des cartes postales. Donc, j'ai l'impression que le " Je" de l'Aquarium est une projection de "l'Autre", ce qui fait qu'il est normal que la fiancée, la promise à l'autre, lui revienne. C'est elle qui fait le lien, raccole le second personnage principal et part avec lui. Et d'ailleurs, dans le fond, ce qui se passe c'est que l'on accède toujours à la vie, que l'on accède toujours à la réalité, à la continuité, par une femme.

A.R. La femme, je crois, dans votre cuvre, est extrêmement importante. Elle est véritablement la salvatrice. 
J.G. C'est elle d'abord qui a permis au Québec d'être ce qu'il est. Non seulement parce qu'elle faisait les enfants et les élevait, mais parce que c'est la seule qui n'ait jamais accepté de compromission. Les hommes ici, les Canadiens français, se sont souvent vendus à l'Anglais, au nom du "réalisme". La femme tenait tête, c'était elle la réalité, c'était elle la langue maternelle, celle qui permet de nommer le monde une première fois. Les hommes s'imaginaient que les objets en anglais existaient plus qu'en français. Nous n'avons pas de prise sur le réel du langage comme la femme.

A.R. Je disais tout de suite qu'Andrée paraît être une co-rédemptrice et vous dites que la femme, peut-être mieux que l'homme, refuse les compromis. Andrée me paraît d'une pureté à ce point de vue-là : elle arrive, on lui apprend que son premier amant a disparu et elle est très spontanée dans son acceptation de l'autre. II y a ces premières scènes qui coïncident avec la cessation des pluies; les personnages vont se purifier, et rappelez-vous, vont dire " on va redécorer".

J.G. Ils refont le décor...

A.R. Refont le décor... après le long sommeil d'Andrée qui est.fatiguée, qui se réveille et qui renaît. Je pense que c'est un éveil plus qu'un réveil: c'est une renaissance. C'est un être nouveau qui naît et qui est prêt, je ne dirais pas à se donner, mais à prendre charge de ce qu'elle fait véritablement. Et c'est elle qui va mener la dynamique de la deuxième partie du roman, jusqu'à ce que les deux personnages se retrouvent.

J.G. Jusqu'au bateau...

A.R. Jusqu'au bateau qui les mène vers New York. J'aimerais que nous parlions, si vous le voulez, de la fin, de la toute fin de ce roman où on invoque l'oubli, c'est-à-dire que l'on vient de quitter un espace où on a vécu, une double expérience en quelque sorte, expérience physique, réelle de ce pays du Tiers-monde et l'expérience (par souvenance): l'expérience canadienne-française. Et l'on part vers New York, et peutêtre vers l'Europe, ça n'est pas sûr. Ce départ est incertain.

J.G. C'est le départ incertain du Canada français : le tiers monde c'était ici, et nous étions tiraillés entre les idées européennes et la technologie américaine. Le bateau part pour New York, ou peut-être est-ce vers l'Europe? Encore aujourd'hui, je ne sais plus combien d'années plus tard, après avoir écrit ce roman, je vois que c'est la même question qui se pose encore et qui se posera pendant des siècles et des siècles, ce poids de l'Europe (l'histoire, la culture, les idéologies) d'un côté, ce poids de l'Amérique (la science, la frontière, l'espace, les techniques) de l'autre.

Tout ce dont on peut être assuré, c'est qu'il s'agit d'un voyage.

A.R. Ça sera un voyage, ça sera un nouveau départ... 
J.G. Ça sera donc l'arrivée quelque part...

A.R. Peut-être une arrivée sans souvenirs parce que le personnage dit: "Andrée il ne faut pas se souvenir". Ce qui veut dire que ce que l'on a atteint de nouveau ne doit pas appartenir à un passé touffu.

J.G. II faut l'inventer!

A.R. II faut véritablement refaire l'existence. Le deuxième personnage de votre cuvre à ce moment-là devient important. Il n'est pas nommé lui non plus, ne sera pas nommé. Pourtant les personnages de son entourage, surtout les femmes, elles, seront nommées. 11 m'intéresse d'autant plus qu'il est doublement fuyard celui-là. II est dans les Prairies, il est à Winnipeg, c'est un évadé des forces armées canadiennes premièrement, et deuxièmement il s'est évadé de Montréal, de son pays natal, de son aire de vie natale, pour vivre à Winnipeg, au Canada anglais, dans ce que le Canada anglais peut représenter, excusez-moi, de plus neutre peut-être à cette époque-là. Ce n'est pas Toronto, ce n'est pas Vancouver, c'est la plaine, c'est l'immensité.

J.G. C'est le cliché aussi! Ce sont ces Rocheuses à proximité dont les fédéralistes nous disent qu'elles nous appartiennent (j'attends le jour où l'on en vendra de petits blocs pour mettre sur les manteaux des cheminées) et c'est le Canada anglais que les fêtes de la Confédération nous ramènent à la surface chaque année. Mais c'est l'ouest aussi. Le Canada anglais, ce ne pouvait être Toronto, qui ressemblait tant à Montréal que le personnage aurait cru changer tout bonnement de quartier. Ce devait être la plaine, les grands espaces, le pays de la police à cheval, celui "where the action is", c'est-à-dire l'argent, le pétrole, aujourd'hui l'Alberta. Le Canada, pour moi, ça n'a jamais été l'Ontario.

A.R. Et Patricia qui est Montréalaise, devient cette représentation d'un autre milieu, d'une autre civilisation, d'une autre race. Et Patricia qui est belle. qui est belle comme femme, qui est jeune, qui est jolie, qui est insoucieuse, peut représenter peut-être la part ludique, l'utopie en quelque sorte.

J.G. Oui. C'est le personnage libre et ensoleillé de la côte ouest. La femme des plages de la baie de Los Angeles. C'est un peu, avant le temps, le mythe de la jeunesse éternelle. Mais nous lisons en ce moment les livres après coup: la réalité rattrape la fiction.

A.R. II y a une chose que j'aimerais soulever et sur laquelle j'aimerais insister un peu avec vous. Ce personnage, on le voit alors qu'il a déjà quitté Montréal, alors qu'il vit avec une jeune femme de langue anglaise (d'une autre perspective sur l'existence) avec laquelle il a un dialogue, à mon avis, très riche au début, dialogue qui va s'atténuer vers la mi-temps de leur existence et qui sera remplacé par des clichés, par des formules genre "You French don't understand, leave me alone with your dreams ", etc. Et tout à coup le personnage éprouvant profondément en 
lui-même le besoin de retrouver ses racines, entreprend ce long périple du retour vers Montréal, par le train. Les deux amis s'installent ensemble d'abord et presqu'immédiatement après il y a la réintégration dans le pays, le pays du Québec. A la fin du roman on va parler du Québec, cette fois-là, et non plus du Canada français. Le pays éclate à l'inté rieur du personnage qui en fait la reconnaissance dans le sens militaire du mot et qui ensuite va renaître lui-même à l'intérieur de son pays grâce à la rencontre qu'il fait avec des amis qui se préoccupent de politique et grâce surtout au personnage de Madeleine, première québécoise qui vient dans votre œuvre. Elle va ensuite mourir de façon accidentelle et violente. C'est un retour important, je pense, dans votre œuvre. C'est le début de la réappropriation.

J.G. C'est une charnière! Tout se passe comme si la première partie du Couteau sur la table était une suite immédiate de l'Aquarium et qu'à un moment, au tiers ou à la demie du livre, il y avait un renversement. Mais ce renversement ce n'est pas seulement le retour en train et le retour à Montréal. C'est aussi le fait que quand j'ai écrit le livre, je l'ai conçu, pensé et commencé comme une histoire d'amour entre une Canadienne anglaise et un Canadien français. Mais pendant que j'écrivais il y eut des jeunes gens qui commencèrent à mettre des bombes dans les boîtes aux lettres et ce terrorisme a radicalement changé l'atmosphère. On est passé de relations entre individus à des relations ramenées à des slogans, à des relations médiatisées, en ce sens que ce sont les média qui ont pris charge. A partir de ce moment-là il n'était plus possible, psychologiquement, humainement parlant, de continuer la même histoire. Ça n'était plus possible pour personne. Donc le livre m'a tourné entre les mains et j'ai tenté de raconter comment tout ça effectivement aboutissait à une brisure. Et c'est ce pourquoi le récit se termine par un communiqué qui est le communiqué du F.L.Q. c'est-à-dire l'insertion de l'actualité dans la vie tranquille, individualisée, calme, amoureuse du personnage principal qui s'imaginait, jusqu'à ce moment-là, Canadien. Personnellement, c'est vers ces années-là aussi que j'ai amorcé un virage politique qui m'était imprévisible.

A.R. Qui vous ètait inattendu?

J.G. Ah! Totalement inattendu! Tout chez moi était fortement orienté vers la culture anglophone, les journaux que l'on recevait à la maison étaient de langue anglaise, les émissions de radio - il n'y avait pas de télévision à l'époque - que nous écoutions étaient américaines, une partie de ma famille habitait vers l'Outaouais, vers le Lake Shore comme on dit ici, le West Island!

A.R. Est-ce que c'était plus que de l'anglophilie. Est-ce que c'était presqu'une appartenance?

J.G. C'était l'appartenance d'abord à une classe politique, qui était la classe libérale, qui n'a pas changé à cet égard; chaque fois que je lis un article 
signé d'un libéral aujourd'hui, j'ai l'impression d'entendre les paroles de mes parents, de mes grands-parents, de mes oncles, de mes tantes, et du milieu auquel j'appartenais. C'était la classe qui croyait que les réalités étaient celles du commerce, d'abord et avant tout, que seul l'Anglosaxon avait une vision juste du monde, que le Canadien français était en soi un être qu'il fallait expulser ou cacher. J'ai donc appris l'anglais, comme la plupart des gens, mais je me suis appliqué à le parler sans accent, c'est-à-dire sans accent canadien-français, ma première tâche étant de passer inaperçu.

Il était normal qu'à ce moment-là ça se passe ainsi. J'avais vingt-cinq ans et je m'en allais dans la direction sûrement aujourd'hui souhaitee par les libéraux. Et puis il y eut ce choc émotif; et cette prise de conscience; grâce à des amis avec qui nous discutions politique; évidemment pas de la façon dont cela est raconté dans le roman. Mais le cheminement dans ma vie fut réel et semblable.

A.R. Est-ce que vous parleriez pour vous, Jacques Godbout, de conversion, de transformation intérieure?.

J.G. Non!

A.R. D'évolution?

J.G. D'évolution normale. C'est un peu ce que nous avions amorcé, les uns et les autres, que ce soit en Europe ou en Afrique, ou ailleurs. Quant à savoir qui nous étions! Nous n'étions certainement pas des Canadiens anglais! Et il était bon qu'on le sache, et qu'on l'accepte, et qu'on le reconnaisse.

A.R. Vous disiez tout de suite "il y a des amis qui ont commencé à me parler politique et j'ai pris conscience". Il y a les amis, il y a sûrement les événements aussi, les bombes, par exemple, dans les années 63,64 .

J.G. II se faisait en nous tous un cheminement profond. II y avait aussi l'aspect symbolique de la révolution algérienne à laquelle nous nous identifions. Les bombes n'ont été, je pense, que l'expression spectaculaire et désespérée de ce cheminement... quand explose un paquet de dynamite on cherche le pourquoi. Mais ces bombes pouvaient nous lancer dans toutes les directions. J'ai des amis de mon âge qui ont plutôt implosé qu'explosé. Ils ont eu honte de nous, du sang sur les mains, ils ont refusé la violence et se sont enfoncés dans le Canada. Les conséquences du terrorisme étaient totalement imprévisibles à l'époque. Nous aurions pu être poussés vers notre mort. Mais le langage, la littérature, m'amenaient dans une autre voie.

A.R. Ça devenait une obligation.

J.G. Oui, c'était un cheminement inévitable. 
A.R. Ça devenait une obligation pour le personnage du Couteau sur la table, par exemple, de briser son anonymat, qu'il ne brise pas tout à fait, remarquez...

J.G. De commencer...

A.R. De commencer à le briser, et je pense que l'éclatement se fera dans Salut Galarneau. La salutation du début du roman est extrêmement importante, à mon avis. Très révélatrice! C'est la somme de tout le roman. Restons un peu encore dans le deuxième roman. J'aimerais que vous continuiez à faire ces associations entre ce qu'était le personnage de vos romans et ce que vous étiez vous-mème: en train d'évoluer très rapidement et de changer le cap, de modifier le cap, de votre propre existence. Vous disiez tantôt et j'avoue que ça m'a un peu surpris, et je trouve que c'est important " $\mathrm{J}$ 'ai appris à parler anglais en n'ayant pas d'accent " pour pouvoir passer inaperçu. C'est peut-être un peu ce que fait le personnage que nous trouvons en début du roman, qui est là-bas dans les plaines et qui passe pour un anglophone?

J.G. Absolument, qui ne doit pas détonner dans le paysage! Mon père m'a raconté que quand il avait vingt, vingt-deux ans, il lui arrivait d'aller se promener rue Saint-Jacques avec un journal anglais sous le bras, le Montreal Star, (et il n'était pas le seul comme ça, disait-il) tout simplement pour que les gens de la rue Saint-Jacques se méprennent alors qu'il parlait à peine leur langue à l'époque. C'était là le comportement de ceux qui vouiaient être acceptés dans une civilisation en marche. Parce que le «Libéral», il faut pas cracher dessus non plus, je parle de ceux qui s'opposaient à la classe politique de l'Union Nationale, le «Libéral» c'était l'urbain, c'était l'avenir.

Moi je suis né à Montréal, et comme mon père était agronome, j'avais la campagne en sainte horreur. II n'était pas pour moi question de redevenir paysan. Le retour à la terre, jamais! Donc il me fallait trouver les assises d'une humanité quelque part; être "libéral", c'était être progressiste, c'était être urbain, c'était donc être Américain. Les films qui formaient, je pense à 80 pour cent, la base même de ma culture, de mon imaginaire, étaient des films américains. Je n'avais pas vu avant l'âge de 18-19 ans un seul film français et quand je les ai vus, je les ai trouvés exécrables. Je n'avais aucun système de références. Je ne comprenais rien à ce qui se passait à l'écran. Je m'étais identifié beaucoup trop longtemps à Humphrey Bogart pour pouvoir croire à ces espèces de personnages ridicules qui se prenaient pour des gangsters dans les rues de Marseille. Pire: ces ombres curieuses parlaient un langage qui m'était étranger! Je crois qu'à ce moment-là, je m'identifiais à l'élément dominant, sans me poser de questions.

A.R. Et Dieu sait que vous allez en parler beaucoup d'américanité. Vous commencez à en parler dans Le couteau sur la table. Déjà d'ailleurs on en parlait un petit peu,mais dans $L e$ couteau sur la table avec toute cette 
espèce de satire de la société de consommation et toutes ces formules qui vous sont chères et qui sont uniques, je pense, dans l'écriture du Québec: "génération Pepsi", "on est des Pepsis", "brossez-vous les dents au Pepsodent». II y a toutes ces séries de poèmes, si vous permettez le mot, je pense que ce sont des poèmes; dans votre intention à vous ce doit être une écriture poétique celle-là, satirique...

J.G. C'est satirique, c'est poétique et ça va beaucoup plus loin. II y a toujours un élément constitutif qui fait en sorte que la culture réunit les gens. Cet élément constitutif a pu être la religion, à un moment donné, qui colorait toute la culture et qui faisait en sorte que les gens avaient des choses en commun, la fuite en Egypte, ou la manne qui tombe à point, ou le vendredi saint qui s'assombrit à trois heures parce que l'on va crucifier Jésus-Christ, et le reste..., or aujourd'hui, qu'on le veuille ou non l'élément constitutif, c'est la publicité. Par exemple, cela saute aux yeux, les publications, les média, se multiplient, à mesure que les publics se subdivisent. Vous pouvez ainsi acheter une revue de sport et une autre de politique mais dans chacune de ces revues, le seul élément que l'on retrouve et qui sera toujours le même, ce sera, par exemple, une page de la Canadian Schenley, une autre vantant la Peugeot $404 \ldots$

A.R. Chez vos personnages cela pousse jusqu'à l'obsession...

J.G. C'est une obsession!

A.R. Ce sont autant d'apparitions, des images fulminantes qui viennent soudain et qui interrompent le cours normal des choses, qui empêchent les gens de penser comme ils le devraient et comme ils le souhaiteraient. J'aimerais, si vous le voulez bien, que nous passions maintenant à ce très beau titre quiest à la mi-temps de votre œuvre, qui s'appelle Salut Galarneau. Cela m'apparaît comme étant une reconnaissance de l'autre, une reconnaissance de l'être québécois, une reconnaissance de soimême. Dès le départ, il y a beaucoup de choses qui sont originales dans ce roman, qui sont nouvelles. Le titre d'abord, bien entendu! C'est la première fois que l'on trouve un nom de famille (il y aura le très beau nom de Michel Beauparlant dont nous parlerons plus loin au moment de $L$ 's/e au dragon). Donc, première fois que l'on a un titre qui devient une salutation, salutation qui devient baptismale, à mon point de vue; c'est comme un baptême.

J.G. Le baptême de personnage principal...!

A.R. Il y a encore l'apparition de la famille, les Galarneau, qui forme un clan, les trois frères, le père que l'on a beaucoup aimé, la mère qui, elle, a gagné l'exil, que l'on vénère presque, qu'on idéalise et que l'on rêve d'aller voir, mais... II y a ce très beau départ: un personnage qui non seulement s'identifie totalement parce que l'auteur l'assume en le nommant, mais qui, pour la première fois devient québécois sans "questionnement". Il est Québécois au point de départ, et, non seu- 
lement se baptise-t-il, mais il se dignifie, il s'ennoblit à partir d'une image américaine, "le Roi du Hot Dog"; il se confère une sorte de dignité dont on n'a pas envie de rire. Personnage qui va voyager, mais autrement que ceux qui l'ont précédé. François voyage grâce à son frère aîné: c'est Jacques qui ira - lui qui porte votre nom - en Europe pour son frère, qui lui fera son éducation sentimentale, grâce aux lettres, son éducation intellectuelle en partie et qui peut-être lui conférera le goût d'écrire. Lui, Jacques, n'écrira plus et, d'ailleurs son personnage va s'estomper dès la rentrée au Canada. II va s'estomper au profit du Québécois, comme si l'on voulait proposer au lecteur une nouvelle image du Québécois, qui n'est plus ce type intellectuel un peu froid, un peu sec, qui est allé en Europe, qui y a appris de belles choses et qui, rentrant, voudrait dominer. Cette victoire me parait importante, cet éclatement de l'être québécois, québécisé, si je puis employer cette expression.

J.G. Ce qui se passe ici, c'est que le "Retour d'Europe" cède la place à celui qui était resté et qui s'assume. Ce qui était probablement très difficile pour moi c'était de voir ces choses et de les accepter après avoir fait le cheminement dont on a parlé plus haut. Me dire «Bon! très bien! $\mathrm{Ce}$ Canadien français que j'ai méprisé en un sens, comme je me suis méprisé moi-même, est-ce qu'il est aussi moche, aussi stupide, aussi paysan qu'on l'a décrit? Et s'il l'est, je le suis aussi! Et si j'arrête de me prendre ou de chercher à me prendre pour un Anglais, si j'arrête de tricher, qui suis-je? Est-ce que je suis capable de voir moi aussi et autour de moi les choses avec humour, avec passion, et de me les approprier? " Mais tout cela s'est révélé difficile à structurer parce que ma première idée, quand j'ai abordé Salut Galarneau!, c'était de placer mon personnage à l'écoute, au centre d'un stand de hot dogs et de frites...

A.R. Au bord de la route, là où les gens passent...

J.G. Là où les autres parlent! Ce devait être comme une suite de conversations tenues devant le stand et où celui qui est devenu Galarneau ne disait pas un seul mot. II écoutait les autres. Mais ça n'a pas tenu trois pages. C'est immédiatement lui qui a pris charge du récit parce que dans le fond, c'était un monde à créer, à inventer et auquel il fallait donner une couleur puis un langage. Qu'est-ce que c'était que ce Québécois ? D'où venait-il ? De quel genre de famille? Quelle vision du monde pouvait-il avoir? Le premier brouillon me décourageait. Ma femme m'a encouragé.

Et puis cela a pris une forme de plus en plus enjouée, puis rapidement c'est devenu un plaisir tellement fantastique, une libération tellement agréable que cela s'est écrit quasi d'un seul trait. La principale partie du livre, disons 80 pour cent du livre, je l'ai écrite dans les vingt jours (enfermé à me faire cuire moi-même des hamburgers et des hot dogs, sans frites parce que c'était trop long). Je découvrais et j'entendais à 
mesure - cela se produisait pour la première fois et je crois que ça ne s'est pas produit depuis - un personnage qui « disait » des choses sous mes yeux. Le parfait dédoublement.

A.R. II y a dans Salut Galarneau une extraordinaire réappropriation du pays, grâce à la route, grâce au stand, grâce aux femmes, grâce à l'écriture, grâce au fleuve, au rite initiatique qui est, nous l'avons souligné plus haut, le baptême de François.

J.G. L'écriture, l'envie de raconter, cela vient par imitation. Le plus souvent un grand-père, une grand-mère peut-être, un adulte de toute façon qui a le temps de parler à l'enfant. Et l'enfant le remercie en devenant écrivain. L'initiation de François n'a pas d'autre sens. Le grand-père donnait accès à l'invention du réel. Le mien avait été hótelier, puis manufacturier, il avait longtemps vécu avec nous, je veux dire chez mes parents, il était riche, il m'avait ouvert des mondes.

Alors en me souvenant de lui j'ai situé le roman dans le pays des Daoust, vers l'île Perrot. Visiblement je m'approchais de chez moi.

A.R. Tout à coup parce qu'il en a marre, que rien ne va plus, que son chemin ne mène nulle part, François s'emmure. Cette claustration, cette idée de claustration, vous est-elle venue spontanément? Cette espèce de voyage autour de ma chambre, à l'intérieur de moi-même...

J.G. Elle est apparue inévitable. On ne contrôle pas les personnages d'un roman comme des marionnettes. Au bout de toute entreprise de création, au bout d'un livre on se retrouve emmuré. Galarneau était seul, il savait qui il était, il lui restait à choisir la vie...

A.R. J'aimerais vous poser une question qui tient à l'anecdote mais qui demeure liée au thème du voyage ou de son contraire... Avez-vous jamais eu l'intention d'abandonner François entre les quatre murs de sa maison?

J.G. Non, je ne crois pas! J'ai dû hésiter parce que je voyais un personnage qui était en train de se défaire, puisqu'à force de vivre seul il finissait par se manger lui-même... Non! Cela n'est pas dans mon caractère. II y a sans doute, fondamentalement, deux attitudes vis-à-vis du problème de la mort. Il y a celui qui, comme Hubert Aquin, passe sa vie à courir après la mort et celui qui s'enfuit afin de l'éviter. Il m'eût été anormal de faire une fin tragique. Le noir m'est une couleur artificielle, je crois qu'il ne m'appartient pas, qu'il ne nous appartient pas...

A.R. Une fin tragique, ça vous aurait peut-être empêché de poursuivre...

J.G. Vous avez sans doute raison, mais cela, je ne le savais pas! Si Galarneau n'était pas monté dans l'échelle pour sauter par-dessus le mur et aller dire aux gens : "voilà, je veux vivre, je veux écrire, je veux vécrire!», je n'aurais pu moi-même continuer. C'était avant tout le besoin d'assumer et sa vie et son manuscrit... 
A.R. Je me permets ici d'ouvrir une parenthèse et de me reporter à André Langevin dont je disais dans un article qu'à la fin du Temps des Hommes, après la sombre trajectoire des personnages d'Évadé de la nuit et de Poussière sur la ville, qu'il se trouvait traqué, réduit au silence, quasi incapable de poursuivre son ceuvre, sûrement pas dans la même veine...

J.G. II lui fallait se débarrasser des murs. Je ne crois pas que le romancier s'en rende compte au moment de l'écriture. Si l'on s'en rendait compte, tout serait plus facile. Mais effectivement il faut se laisser une porte ouverte si l'on veut continuer d'écrire.

A.R. Jacques Godbout, j'aurais envie de vous demander maintenant quelle transition vous faites entre Salut Galarneau et D'Amour P.Q. Je veux savoir ce qui existe dans l'entre-deux romans.

J.G. Un film important pour moi: le roman d'IXE-13 en comédie musicale. C'est-à-dire l'ironie complice à propos de notre culture ti-pop. Dans ce film il n'y a qu'une image réelle, c'est celle de l'église. Tout le reste n'est que clichés en couleurs, et symboles de nos aspirations. Par la suite j'ai eu besoin d'écrire un livre qui allait tracer le bilan du trajet poursuivi de l'Aquarium à Salut Galarneau! J'ai eu envie d'en finir aussi avec le côté sacro-saint du créateur. J'avais envie d'être heureux. Et de dire clairement que celui que j'étais avait été pensé, inventé, et produit par la femme.

Dans D'Amour P.Q. les deux secrétaires prennent charge de l'univers de l'écrivain. Elles sont à l'aise dans leur peau. Les problèmes de l'écrivain ne faisaient pas le poids, devant la vie, et je croyais que le Québec allait exploser enfin!

A.R. Et c'est curieux, la femme prend d'abord charge de l'écriture. Bien sûr elle se prend en charge elle-même; elle me paraît très épanouie dans son corps, dans son âme, dans tout son être; elle est belle, elle le sait, elle en profite. Je demeure heureusement surpris qu'elle prenne charge de l'acte d'écrire, du voyage de l'écriture, de cette aventure fondamentale qui est recherche et errance...

J.G. C'est évidemment un petit péché d'écrivain de croire que l'acte d'écrire est fondamental; probablement que l'ingénieur dirait que l'acte fondamental, c'est celui de bâtir un pont! (Pontifex. Le grand pontife!) Mais je soupçonne cependant qu'il y a dans l'écriture quelque chose de plus essentiel, de plus mythique que dans la construction d'un pont, parce que le langage va chercher dans tout ce qui est nous, dans tout ce qui est ancien, le nouveau et le désir. Donc le fait d'assumer l'écriture, c'était aussi le fait d'assumer la vie, c'était de s'entendre dire: « Monsieur l'écrivain pour qui vous prenez-vous? Essayez donc de cesser de croire que votre roman est autre chose que l'excroissance plus ou moins phallocratique de vos aspirations. Ce n'est pas parce que vous écrivez que l'on doit se mettre à genoux devant vous. Vous avez le droit d'écrire, 
nous aimons ce que vous faites, mais ça n'est pas non plus la fin du monde. Ensemble nous allons faire l'histoire du roman au Québec. Le premier roman que vous me présenterez ressemblera à ce qui s'écrivait dans les années cinquante, un peu ampoulé, avec une histoire biblique, bien entendu, puisque c'était l'Histoire de l'époque, et peu à peu nous allons transformer tout cela pour en faire un récit moderne, contemporain. De roman en roman jusqu'à Radio-Canada, où dans une émission du réseau M.F. l'on fera le tour du livre et de ses cérémonies...

A.R. C'est ainsi que l'on voyage de la mythologie d'hier à celle d'aujourd'hui, d'une forme d'écriture à une autre, d'un moyen de communication à un autre, d'une langue, enfin, à une autre. Entre l'écriture de l'Aquarium et celle de D'Amour, il y a toute une différence. D'Amour est de conception plus téméraire, se rapproche de l'image, de la succession de séquences, peut-être de la bande dessinée...

J.G. D'Amour P.Q. est un livre que j'ai écrit comme si je l'avais vu à la télévision. La télévision, c'est du cinéma sans aucune des subtilités que permet le $35 \mathrm{~mm}$. La télévision est au cinéma ce que la bande dessinée est au récit d'aventure. Mais le dessin m'a toujours plus intéressé que la couleur. J'ai toujours préféré Picasso à Juan Gris. Il y a plus d'efforts de perspective dans les bandes dessinées parfois que dans des tableaux de la Renaissance. Oui, enfin, Mireille et Mariette font une espèce de récit moderne, avec notre vieux fonds culturel, enfantin et riche tout à la fois.

A.R. D'une mythologie très contemporaine!

J.G. C'est cela! Elles s'amusent, elles sont spectaculaires, théâtrales. C'est une pièce de théâtre, puisque nous en sommes réduits à un lieu fermé, Côtes-des-Neiges, c'est chez moi! Il s'agit d'un voyage mental, sur une scène autour d'un lit, dans un lit, dans un pays conquis, c'est-à-dire qui appartient à ceux qui l'habitent enfin.

A.R. Ni Mireille ni sa compagne ne se posent de questions sur leur appartenance, ni sur leur passé, ni sur leur avenir, ni sur leur langage; elles sont préoccupées par leur présent dont elles profitent pleinement.

J.G. Oui, petit message, en passant, aux gens de ma génération. C'est que pour ceux qui ont moins de trente ans, moins de 25 , ces problèmes de conquête, de reconquête du Canadien français, de son double, de son triple n'existent pas. Toutes choses vont de soi. Ces gens sont à l'aise chez eux, dans leur peau et dans leur langage.

A.R. II n'y a plus d'anxiété!

J.G. S'il y en a, cela n'est plus la même!

A.R. Et pourtant le personnage de L'/s/e au dragon redevient voyageur et problématique et sans doute aussi un peu anxieux. 
J.G. A l'époque où j'écrivais D'Amour P.Q. j'avais l'impression que les gens autour de moi avaient pris charge de leur vie, de leur monde et puis qu'on avançait. Or durant les quatre ou cinq premières années de 1970 nous avons vécu une débandade culturelle. Il s'installa même une morosité dont Bourassa a fait les frais finalement. Je n'ai pas échappé moi-même à ce découragement, ce n'était pas un désespoir, seulement une désespérance. Celle des résistants qui parfois se demandent si cela vaut la peine de résister. Et puis m'est apparu le personnage de Beauparlant, qui était au fond aussi bien Gilles Vigneault que Réjean Ducharme, c'est-à-dire celui qui veut que tous se tiennent debout, pour giguer ou pour crier, qu'importe, mais au moins pour tenir un discours digne. Dans la désespérance Beauparlant se dit qu'il n'a pas planté un chêne pour perdre sa peine, il est prêt à parler dans le vide du fleuve, à lancer ses bouteilles à la mer, même si personne ne les ramasse, même si personne ne répond.

Mais Beauparlant a une crainte, celle de radoter. C'est sans doute la mienne aussi, radoter c'est devenir sénile, raconter toujours les mêmes histoires et sans s'en rendre compte ne plus intéresser, devenir ennuyeux. Plutôt mourir!

Nous sommes d'une génération qui a aimé faire des discours, puisque nous appartenons à un clergé séculier qui a remplacé l'autre...

A.R. Vous l'avez baptisé, celui-là, Beauparlant, c'est-à-dire, je suppose, le bien parlant?

J.G. Oui, celui qui parle, celui qui parle bien. Celui d'ailleurs qui ne fait que parler et qui agit fort peu sinon par la magie. C'est un conte, il habite luimême le conte. Tout cela s'est construit d'une façon un peu inattendue. C'est un livre qui est venu comme tous les autres, des circonstances, des choses que j'ai vues, des choses que j'entendais autour de moi. J'ai eu une aventure personnelle avec des gens d'une Compagnie étrangère à l'Île Verte près de Rivière-du-Loup. Ces gens voulaient y stocker du pétrole. J'ai mené ma petite bataille et aú moment où je croyais qu'ils allaient gagner, les Arabes du jour au lendemain ont décidé d'augmenter le prix du pétrole et leur projet s'est effondré. Qui dit Arabe, pour moi, dit Bagdad, dit Contes des mille et une nuits. Ça m'a sauté aux yeux : il n'y avait qu'une façon de parler des Beauparlant effectivement et c'était en invoquant la magie. Le conte. De là l'île investie par le Dragon, lequel est à la fois mythe nécessaire, et une mystification dont on doit se débarrasser. Mais surtout celui qu'il ne faut jamais tuer.

A.R. Surtout pas. Parce que le Dragon est protecteur. C'est une très belle image et qui me paraît exceptionnelle dans votre roman. Cela vous permet de vous élever au-dessus de l'histoire immédiate - malgré les sources d'inspiration - pour atteindre à la féérie, à l'affabulation.

J.G. Cela fait partie de la mythologie du Bas-du-fleuve. Là-bas, comme dans mon livre, plusieurs personnes ont vu des dragons, ce qui veut dire qu'il 
y en a peut-être bien. Les animaux fabuleux, si on remonte aux textes des découvreurs, sur les cartes...

A.R. Champlain lui-même en a vu au moins un...

J.G. Bon! Si Champlain en a vu, c'est qu'il y en avait.

A.R. Oui, dans un récit de voyage, dans une lettre, on fait allusion à un monstre marin surgissant des profondeurs et effrayant tous les témoins... Et cette lettre est sérieuse...

J.G. Je suis très sérieux moi aussi!

A.R. Nous n'avons parlé ici ni de l'œuvre critique, ni de l'œuvre cinématographique. Nous aurons, je le souhaite, l'occasion de poursuivre cet entretien. Je veux revenir à l'œuvre romanesque pour vous demander si vous en voyez le devenir...

J.G. Hier, j'ai atteint une étape assez merveilleuse... Je ne sais pas encore ce qui va en résulter.

J'avais depuis huit mois commencé l'écriture d'un autre roman. La première période de gestation m'a donné dans les 80 ou 100 pages. Cela s'est terminé fin février. Mais j'ai tout relu et puis trouvé que ça n'avait aucun intérêt. C'était trop prosaïque, trop proche de la réalité. Ça se passait en Haïti ; ça devenait un documentaire, en bonne partie. Alors j'ai mis de côté ce brouillon en me disant qu'un jour j'y reviendrais...

Puis j'ai remis le collier quelques semaines plus tard en profitant d'un long séjour en Europe pour avoir la paix et écrire dans le silence. Et je suis revenu à Montréal et j'ai tout relu à nouveau. Et puis j'ai découvert que ce deuxième brouillon était lui aussi merdeux et sans intérêt. Et j'ai repris toujours sans me décourager parce que je sais que les débuts sont ainsi... Jusqu'à ce que la mayonnaise prenne, on ne sait jamais si on a mis une goutte d'huile en trop! Enfin, ce qu'il faut, c'est que la mayonnaise prenne. Alors, j'ai recommencé avec un peu de moutarde dans le fond de l'assiette puis un jaune d'œuf, et puis l'huile et un peu d'ail et puis tout à coup, hier, je me suis rendu compte que j'en étais à ce moment privilégié (pour un écrivain québécois) où il peut dire: “Qu'ilsen-mangent-donc-toute!». Ça, c'est le moment précieux où l'on cesse d'écrire en pensant aux quatre ou cinq critiques qui ont dit ceci ou cela, précédemment, sur les autres romans; quand on cesse de penser aux professeurs, aux groupes d'élèves qu'on a rencontrés et qui étudient ou lisent ou analysent nos livres; quand on élimine les amis qui gentiment vous disent: "As-tu quelque chose sur le métier?"; quand on réussit à laisser tomber dans l'oubli les confrères écrivains dont la moitié probablement se dit: " Pourvu qu'il écrive un bon livre, cette fois-ci! ». Et l'autre moitié: "Pourvu qu'il se casse la figure!". Quand tout ça passe, quand on comprend qu'on n'est "surveillé" que par un aéropage imaginaire - lors de mon premier livre personne ne me surveillait, ça 
allait bien - quand on réussit à se débarrasser de toutes ces personnes, qui ne savent même pas leur poids, et vous épient...

J'ai donc mis presque six mois à me débarrasser de ces gens et quand je suis arrivé, hier, au moment de les envoyer au diable j'ai enfin compris que je pouvais faire désormais un livre pour mon plaisir! Qu'il soit mauvais, qu'il soit excellent, qu'il soit beau ou laid, qu'il puisse se relier aux cinq précédents ou pas, je m'en fous! Ce sera leur problème et non plus le mien! Dès ce moment j'ai pu recommencer à écrire assez librement. Mais ça ne fait que quelques heures que je suis dans cet état. Je regarde de très près les deux personnages qui se sont rencontrés sur une plage de mon cahier hier, et dont je ne sais pas du tout où ils s'en vont aller. J'en suis au point où il faut que l'un des deux porte un nom... Je suis embêté parce que le nom qu'on donne, d'une certaine manière, donne à son tour une allure au personnage... Dans quelques pages je devrai aussi lui trouver un état civil et quand il aura eu un nom et un état civil, il aura reçu quelque part une éducation, un système de références et puis ce personnage va diriger la circulation des mots...

A.R. II va prendre vie et chair...

J.G. Oui, et il ne me laissera plus autant de latitude, de liberté que j'en ai maintenant. Ni de possibilités! Or le moment où tout commence est fantastique puisque tout est possible! C'est comme aborder une inconnue! Le comble de l'érotisme!

A.R. Avez-vous l'habitude de vous sentir opprimé par vos personnages, au moment où ayant pris chair et mouvance ils imposent leurs choix et leurs aspirations et leurs propres besoins.

J.G. Pas opprimé, mais dirigé hélas oui! II y a certains gestes qu'ils ne peuvent plus faire, puisqu'ils ont atteint tel âge... Eh! Si le bonhomme boite, il ne peut pas courir... on ne savait pas, quand an a commencé à le faire boiter, vers la page 10, qu'il aurait besoin de courir comme un chien à la page 50 et qu'il tomberait victime du sadique! L'auteur est pris avec tout ça, qui fait partie du plaisir de la constitution de l'écriture en opposition à la réalité, jusqu'à ce qu'elle en devienne une à son tour. Ce qui va m'amuser dans les semaines et les mois qui viennent, ce sera de voir ce qui adviendra du récit, maintenant que j'ai atteint le stade de la mort des censeurs imaginaires, les lecteurs, mes frères... 